\title{
Blood cell histology of horseshoe crab, Tachypleus gigas.
}

\begin{abstract}
Amoebocytes are the main carrier of innate immune system in horseshoe crabs. Cytoplasm of these cells are packed with granules which consist of all coagulation factors and anti-bacterial compounds. Light microscopy (using May-Grunwald and Giemsa staining technique) of 46 blood specimen for both sexes of Tachypleus gigas and observation under Transmission Electron Microscopy (TEM) were performed. Light microscopy revealed that these are ovoid cells containing blue nucleus surrounded by a cytoplasm packed with pink granules. Large and small axes measured $18.8 \pm 0.5$ and $12.8 \pm 0.4 \mu \mathrm{m}$, respectively. Total blood cell count (33000 cell mm-3) does not show any significant difference between sexes. TEM of normal and degranulated blood cells revealed that $\mathrm{T}$. gigas hemocytes consist of two type of granules (large and small). Blood cells have all the necessary organelles of normal cells. During the granule formation, fusion of different intermediate granules were observed. Degranulated hemocytes under light microscope and TEM showed cells containing large nucleus and cytoplasmic granules were replaced with a large cytoplasmic vacuole. In light and electron microscopy only one type of hemocyte was observed, the granular cells. These granular cells consist of large and small granules.
\end{abstract}

Keyword: Amoebocytes; Granular blood cell; Histology; Horseshoe crab; Tachypleus gigas. 